\title{
A Beginners Guide to: Aberration Corrected Electron Microscopy
}

\author{
David C. Bell \\ School of Engineering and Applied Sciences, Harvard University, Cambridge, MA
}

The tutorial is for anybody that wants to know something about aberration corrected electron microscopy. Aimed at a very introductory level topics covered will include the background, basic theory, implementation and applications of aberration corrected optics in TEM and STEM. Aberration corrected microscopy has been at the frontiers of materials and biological research for over a decade and is becoming a mainstream concept in the contemporary electron microscopy instrumentation [1\&2]. Though the fundamentals of the theory of aberration correction are well understood, the investigation of novel materials is posing new challenges and driving further improvements in the corrector technology and its integration within the electron microscope. Of a particular interest to scientists is not just the ability to significantly improve overall imaging resolution (currently in the $0.5 \AA$ range) but the ability to significantly improve imaging resolution in the low voltage regime (sub $80 \mathrm{kV}$ ). Such improvements in aberration corrected instruments will further advance the ability to image beam sensitive materials with atomic level resolution and minimal damage, which has not been possible until now.

We will cover origin and types of aberrations, their effects on contrast and resolution and current strategies for correction of axial and field aberrations. Current design of aberration correctors will be discussed as well. We will show how presence of aberrations impacts atomic-resolution TEM and STEM imaging and how the resolution of the images can be significantly improved by employing an aberration corrector. Design and additional benefits of adding a monochromator and an energy filter to an aberration corrected microscope will be addressed. Various examples of current instrumentation options will be highlighted along with a variety of application examples, especially focusing on improvements in low voltage performance (Fig. 2) and microanalysis characterization (EDS and EELS).

This tutorial will be recorded via a lecture capture system and made available to MSA and MAS members at no charge on the MSA website (http://www.microscopy.org)

[1] P.W. Hawkes, Advances in Imaging and Electron Physics, Volume 153: Aberration-corrected microscopy, (2008).

[2] P.W. Hawkes and J.C.H. Spence, Science of Microscopy, (2009). 


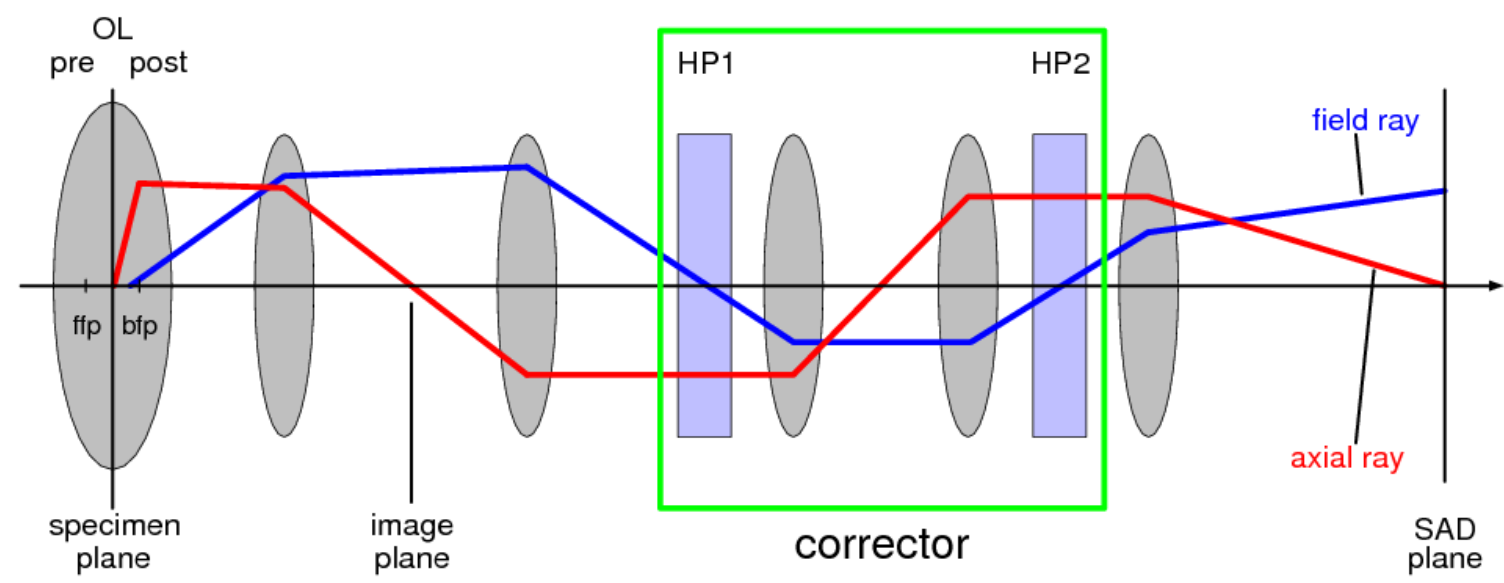

Fig. 1 Example of a design of spherical aberration corrector from CEOS (Courtesy of Max Haider).
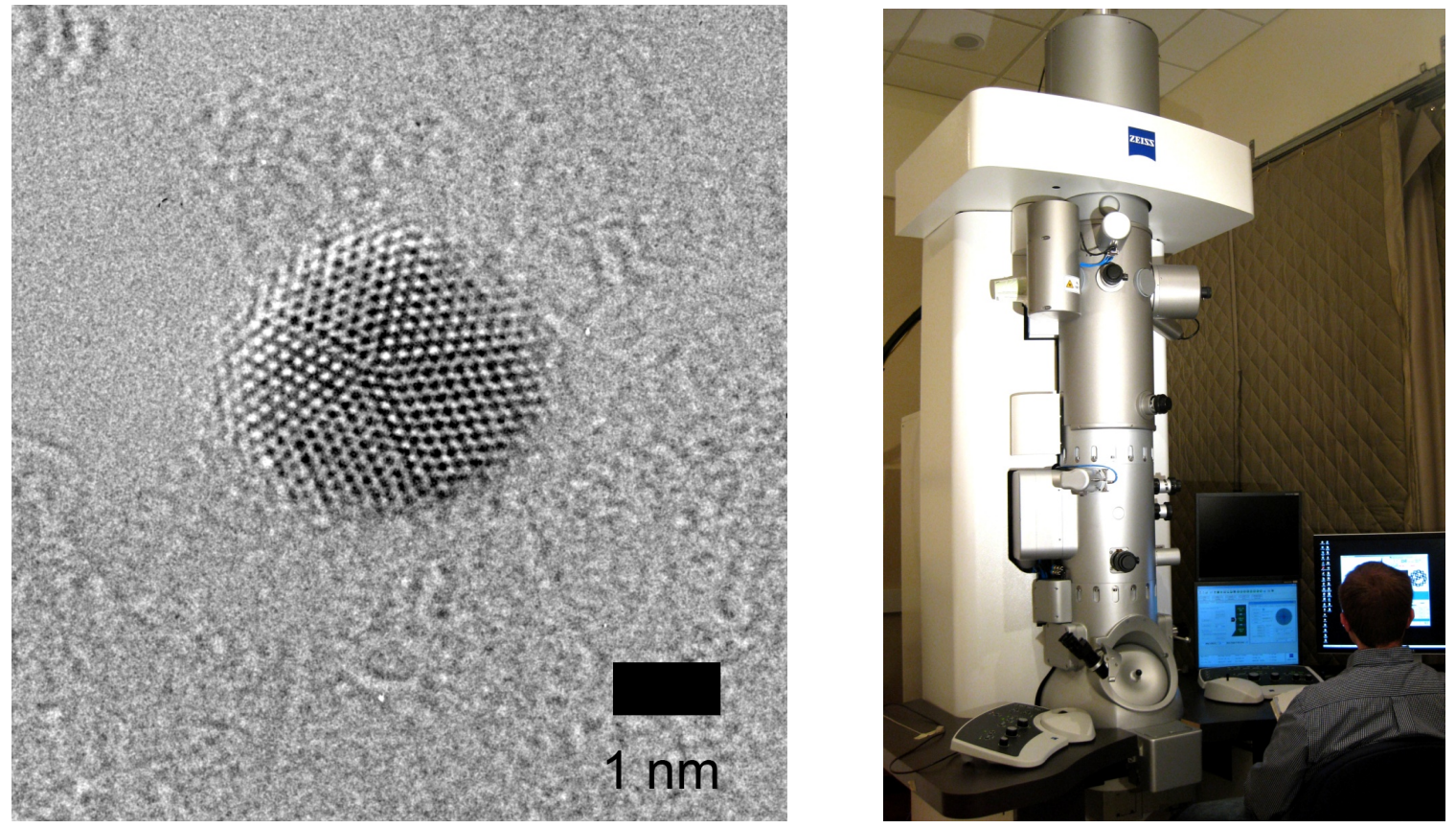

Fig. 2 Left, Single crystal gold nanoparticle imaged at $80 \mathrm{kV}$ with Cs correction and monochromation $\Delta \mathrm{E}=0.1 \mathrm{eV}$. Right, a modern 200-80kV aberration corrected Microscope 